\title{
A NOTE ON THE JACOBSON RADICAL
}

\author{
YU-LEE LEE
}

(Communicated by Maurice Auslander)

\begin{abstract}
In this paper we solve a problem of Divinsky to represent the Jacobson radical as a lower radical class.
\end{abstract}

The purpose of this paper is to represent the Jacobson radical as a lower radical class.

For each class of rings $M$, there exists a radical class $U(M)$, called the upper radical class determined by $M$, such that $U(M)$ is the largest radical class with every ring in $M U(M)$-semisimple [2, 5, 8]. For each class of rings $L$, there exists a radical class $Y(L)$, called the lower radical class determined by $L$, such that $Y(L)$ is the smallest radical class containing $L[1,3,4,6-$ 8]. It is clear that every radical class is the lower radical class determined by itself; the Jacobson radical can be characterized as the upper radical of the class of all primitive rings $[1,7,8]$. Divinsky raised the problem of identifying the Jacobson radical as the lower radical of some reasonable class of rings $[1,8]$. We have the following solution.

Theorem 1. Let $J$ be the Jacobson radical class. For each ring $A$, let $J(A)$ be the Jacobson radical of $A$ and $J_{2}=\left\{(J(A))^{2}: A\right.$ is a ring $\}$. Let $N$ be the class of all nilpotent rings. Then the Jacobson radical class is the lower radical class determined by $\mathrm{N}$ and $J_{2}$.

Proof. It is clear that $Y\left(J_{2} \cup N\right) \subseteq J$. Let $A \in J, I \supsetneqq A$ and $A / I$ be any nonzero quotient ring of $A$. If $I \supseteqq(J(A))^{2}$, then $A \supsetneqq(J(A))^{2}$ and $A / I=\left(A /(J(A))^{2}\right) /\left(I /(J(A))^{2}\right)$. We have $\left(A /(J(A))^{2}\right)^{2}=\left(J(A) /(J(A))^{2}\right)^{2}=$ 0 . Hence $A /(J(A))^{2} \in N$ and $0 \neq A / I \in N$.

If $I \nsupseteq(J(A))^{2}$ then $A / I \geq I+(J(A))^{2} / I \cong(J(A))^{2} / I \cap(J(A))^{2} \neq 0$.

$$
\begin{aligned}
(J(A))^{2} / I \cap(J(A))^{2} & =\left(J(A) / I \cap(J(A))^{2}\right)^{2} \\
& =\left[J\left(A / I \cap(J(A))^{2}\right)\right]^{2} \in J_{2} .
\end{aligned}
$$

Hence we have that $A \in Y\left(J_{2} \cup N\right)$.

Let $S$ be any radical class and $S_{n}=\left\{(S(A))^{n}: A\right.$ is a ring $\}$. By the same proof, we have the following more general result.

Received by the editors March 29, 1991 and, in revised form, September 24, 1991.

1991 Mathematics Subject Classification. Primary 16N20, 16N80.

Key words and phrases. Jacobson radical, lower radicals. 
Theorem 2. If $S$ is a radical class containing all nilpotent rings $N$, then $S$ is the lower radical class determined by $N$ and $S_{n}$ for any positive integer $n$.

We wish to give an example to show that $N \nsubseteq J_{2}$.

Example. Let $A$ be the ring generated by $\{a\}$ with $2 a=a^{3}=0$. Then $A$ is a nilpotent ring. If $A \in J_{2}$ then there is a ring $B \in J$ with $A=B^{2}$ and $B$ is nilpotent. Suppose that there exists $b$ in $B$ such that the ideal $C$ generated by $\{A, b\}$ satisfies $C^{2}=A$. Then $b^{2} \neq a$; otherwise, $b^{3}=a b \notin A$. Also, $b a \neq a, b a \neq a^{2}+a, a b \neq a, a b \neq a^{2}+a, b a^{2} \neq a, b a^{2} \neq a^{2}+a, a^{2} b \neq a$, and $a^{2} b \neq a^{2}+a$. For if $a=b a$ then $a=b^{n} a=0$. The other inequalities can be verified in a similar way. Hence $b^{2}=a^{2}+a$. Now $b a \neq 0$; otherwise, $0=b^{2} a=a^{3}+a^{2}=a^{2}$. Hence $b a=a^{2}$ and $b^{2}=a^{2}+a=b a+a, a=b(b-a)$, and $a^{2}=b(b-a) a=b\left(b a-a^{2}\right)=0$. Hence $C^{2} \nsubseteq A$ and $C^{2}=\left\{0, a^{2}\right\}$.

Hence there are $b$ and $c$ in $B$ such that the ideal $D$ generated by $\{A, b, c\}$ satisfies $D^{2}=A$, while the square of the ideal generated by $A$ and $b$ or $c$ is $\left\{0, a^{2}\right\}$. If $b c=a$ then $c a=a^{2}$; otherwise, $c a=0$ and $0=b c a=a^{2}$. However, $a^{2}=b c a=b a^{2}=b^{2} a^{2}=b^{n} a^{2}=0$ and hence $b c=a+a^{2}$. Then $c a=a^{2}$; otherwise, $c a=0, a^{2}=\left(a+a^{2}\right) a=b c a=0$. However, $a^{2}=\left(a+a^{2}\right) a=b c a=b a^{2}=b^{n} a^{2}=0$; which is a contradiction. Similarly $c b \neq a$ and $c b \neq a^{2}+a$. Hence $D^{2} \varsubsetneqq A$ and $B^{2} \varsubsetneqq A$ and $A$ is not in $J_{2}$. Therefore $N \nsubseteq J_{2}$ and $J_{2} \neq J$.

This example also shows that $N \nsubseteq S_{n}$ and $S_{n} \neq S$ for any radical class $S$ containing all nilpotent rings and $n \geq 2$. Because of the lack of a mechanism, it is tedious to verify a ring not in $J_{2}$. It would be interesting to find more examples of rings in $J \backslash J_{2}$ and to establish general properties.

\section{ACKNOWLEDGMENT}

The author wishes to thank the referee for valuable suggestions.

\section{REFERENCES}

1. N. J. Divinsky, Rings and radicals, Univ. of Toronto Press, Ontario, 1965.

2. B. J. Gardner, Radical theory, Longman Scientific and Technical, England, 1989.

3. A. G. Kurosh, Radicals of rings and algebra, Mat. Sb. 33 (1953), 13-26; English transl., Rings, modules, and radicals, Colloq. Math. Soc. János Bolyai, vol. 6, North-Holland, Amsterdam, 1973, pp. 297-312.

4. W. G. Leavitt, The general theory of radicals, Univ. of Nebraska, Lincoln, NE, 1972.

5. Y. L. Lee, On the construction of upper radical properties, Proc. Amer. Math. Soc. 19 (1968), $1165-1166$.

6. __ On the construction of lower radical properties, Pacific J. Math. 28 (1969), 393-395.

7. F. A. Szasz, Radicals of rings, Wiley, New York, 1981.

8. R. Wiegandt, Radical and semisimple classes of rings, Queen's Univ., Kingston, Ontario, Canada, 1974.

Department of Mathematics, Kansas State University, Manhattan, Kansas 66506 